\title{
Previous and First Detected Cardiovascular Diseases in Patients with New Coronavirus Pneumonia: Possible Mechanisms and Place in a Unified Prognostic Model
}

\author{
Olga V. Blagova ${ }^{a}$ Nadezhda V. Varionchik ${ }^{a}$ Vladimir A. Zaidenov ${ }^{b}$ \\ Polina O. Savina ${ }^{a}$ Natalia D. Sarkisova ${ }^{a}$

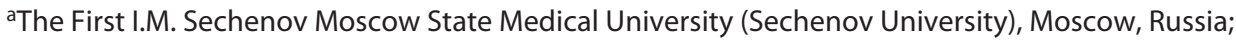 \\ ${ }^{b}$ Moscow City Clinical Hospital No. 52, Moscow, Russia
}

\section{Keywords}

Coronavirus pneumonia - Coronavirus disease 2019.

Anti-heart antibodies $\cdot$ Myocarditis $\cdot$ Prognosis

\begin{abstract}
Purpose: The aim of this study is to evaluate the frequency of cardiac involvement in patients with coronavirus disease 2019 (COVID-19), possible immune mechanisms of myocardial injury, and the place of cardiovascular pathology among other prognostic factors. Methods: The study included 86 patients (48 male, $60.2 \pm 16.6$ years) with COVID-19. In addition to common investigation, examination of troponin $T$ $(n=18)$ and anti-heart antibodies (AHA, $n=34$ ) were used. The average hospital period was 14 [12; 18] days. Results: The incidence of cardiovascular disease and symptoms was $45.3 \%$. Arrhythmias, heart failure, low-QRS voltage, repolarization disorders, and pericardial effusion were the typical for coronavirus cardiac injury. The level of AHA was increased in $73.5 \%$. Significant $(p<0.05)$ correlations of AHA level with inflammatory activity, pneumonia, respiratory failure, cardi-
\end{abstract}

karger@karger.com www.karger.com/iaa

Karger $\stackrel{\text { ' }}{5}$

BOPEN ACCESS
(C) 2021 The Author(s)

Published by S. Karger AG, Basel

This is an Open Access article licensed under the Creative Commons Attribution-NonCommercial-4.0 International License (CC BY-NC) (http://www.karger.com/Services/OpenAccessLicense), applicable to the online version of the article only. Usage and distribution for commercial purposes requires written permission. ac symptoms, and death were found. D-dimer $>0.5 \mu \mathrm{g} / \mathrm{mL}$ had a sensitivity of $79.2 \%$ and specificity of $60 \%$ in the prediction of cardiovascular manifestations. Cardiac failure was one of the causes of death in $3 / 8$ patients (37.5\%). Lethality in the presence of cardiovascular pathology was 17.9 versus $2.2 \%$ without it, $p<0.05$. The most powerful prognostic model includes age, diabetes, oxygen therapy volume, maximum leukocyte level, C-reactive protein, and D-dimer (correlation coefficient $0.871, p<0.001$ ). The model with only age, diabetes, and cardiovascular disease included also had predictive power (correlation coefficient 0.568, $p<0.001$ ). Conclusions: The cardiovascular pathology is frequent in patients with COVID-19 and strong correlates with the D-dimer. It indicates the high significance of prothrombotic and ischemic mechanisms. High AHA levels may reflect an inflammatory heart injury. The cardiovascular pathology is associated with higher lethality.

(C) 2021 The Author(s) Published by S. Karger AG, Basel

Edited by: H.-U. Simon, Bern.
Correspondence to:

Olga V. Blagova, blagovao@mail.ru 


\section{Introduction}

The new coronavirus infection caused by the SARS$\mathrm{CoV}-2$ virus (severe acute respiratory syndrome-related coronavirus 2), called coronavirus disease 2019 (COVID-19), has placed all physicians around the world before a completely new reality. One of the most remarkable characteristics of COVID-19 is a pronounced immune-inflammatory response (hyperinflammation and cytokine storm), which determines unfavorable outcomes [1].

The spectrum of inflammatory and immune reactions includes increasing levels of interleukins $1,2,6,7,10,12$, 18 , tumor necrosis factor alpha, gamma interferon, VEGF, and other biomarkers [2]. On the other hand, patients with a severe course of COVID-19 have lower CD4+ and CD8+ T-lymphocytes, which makes the SARSCoV-2 similar to the human immunodeficiency virus [3]. These factors produce a specific pattern of organ lesions. Cardiac injury caused by a high number of myocardial angiotensin convertase 2 (viral receptor), hypoxemia-induced oxidative stress, and microvascular pathology [4] is detected in $4.8-12-19.7 \%$ of patients with COVID-19 $[2,5,6]$.

Cardiac involvement significantly impairs the prognosis. In Wuhan, myocardial injury was the cause of death in $7 \%$ of cases and its combination with respiratory failure in another 33\% [7]. The analysis of $>72,000$ cases showed: the total lethality was $2.3 \%$, in hypertensive patients, this value increased to $6.0 \%$, and in patients with all cardiovascular disease - up to $10.5 \%$. It was higher than in diabetes, cancer, chronic respiratory diseases [8].

Influence on the prognosis has both previous heart disease and acute injury within the coronavirus infection itself. Scenarios (mechanisms) of cardiac injury include the myocarditis (both direct viral and virus-negative lymphocytic), low-grade inflammation in the presence of a virus in the myocardium, lesions due to generalized endotheliitis, and caused by cytokines and autoantibodies [9]. These scenarios suggest a strong association between cardiac affection and overall disease activity.

However, the specific mechanisms of myocardial injury are not yet fully understood. In particular, the level of anti-heart antibodies (AHA) was not studied in COVID-19. The input of cardiac injury to the prognosis and its correlation with other factors are not fully clear. This makes the formation of a unified predictive model actual, including the heart involvement as a component.

\section{Purpose}

The aim of this study is to evaluate the frequency of cardiac involvement in patients with COVID-19, possible immune mechanisms of myocardial injury, and the place of cardiovascular pathology among other prognostic factors.

\section{Methods}

The Patients Included in the Study

The study included 86 patients ( 38 female and 48 male, average age $60.2 \pm 16.6$ years, from 20 to 90 years) who were treated in Sechenov University Hospital for moderate and severe COVID-19 in April-June 2020.

\section{Inclusion Criteria}

Age over 18 years, presence of typical (according to CT data) coronavirus pneumonia, in combination with the typical clinical symptoms, patient informed consent. Diagnostics and severity of COVID-19 and volume of pneumonia by CT were assessed according to the Guidelines of the Russian Ministry of Health dated April 24, 2020 (version 6).

\section{The Exclusion Criterion}

The exclusion criterion (only for evaluation of AHAs) was an acute coronary syndrome that occurred $<3$ months before hospitalization or during follow-up in patients with verified coronary atherosclerosis (stenoses over 50\%).

\section{Methods of Investigation}

The diagnosis was confirmed in 51 patients (59.3\%) by repeated PCR of nasopharyngeal smears for SARS-CoV-2. In other cases, the diagnosis of COVID-19 was based on the following criteria: contact with a serologically confirmed COVID-19 patient within 2 weeks before clinical manifestation, persistent fever of at least $38^{\circ} \mathrm{C}$, typical CT findings (bilateral “ ground glass opacity”), cough, transient smell and taste disappearance, and typical changes in blood tests in the absence of evidence for other infectious diseases. Both serologically confirmed and unconfirmed types of COVID-19 are present in the International classification of the diseases and have codes U07.1 and U07.2, respectively.

In all patients, clinical and biochemical blood tests for C-reactive protein (CRP), fibrinogen, $\mathrm{Cr}$, glucose, ferritin, aspartate aminotransferase, alaninaminotransferase, Cr kinase (CK), lactate dehydrogenase (LDH), D-dimer, international normalized ratio (INR), also pulse oximetry, electrocardiography (ECG), and chest CT were performed. In addition, examination of troponin T $(n=18)$, interleukin-6 $(n=4)$, prepsin/procalcitonin $(n=4)$, AHA $(n=34)$, and echocardiography (EchoCG, $n=17$ ) was used. The study of AHA was carried out by immunoassay with the use of pure antibodies against human IgG, labeled FITC (fluorescein isothiocyanate), and luminescent serums against human IgG. Antibodies to the nuclei of cardiomyocytes, endothelial antigens, cardiomyocytes, smooth muscle, and conductive system fibers were determined. The patients were selected for this study by random method.

\section{Therapy and Outcomes}

Treatment of a coronavirus infection was prescribed according to Temporary recommendations of Ministry of Health of Russia and regulating orders on COVID-hospital of Sechenov University. 


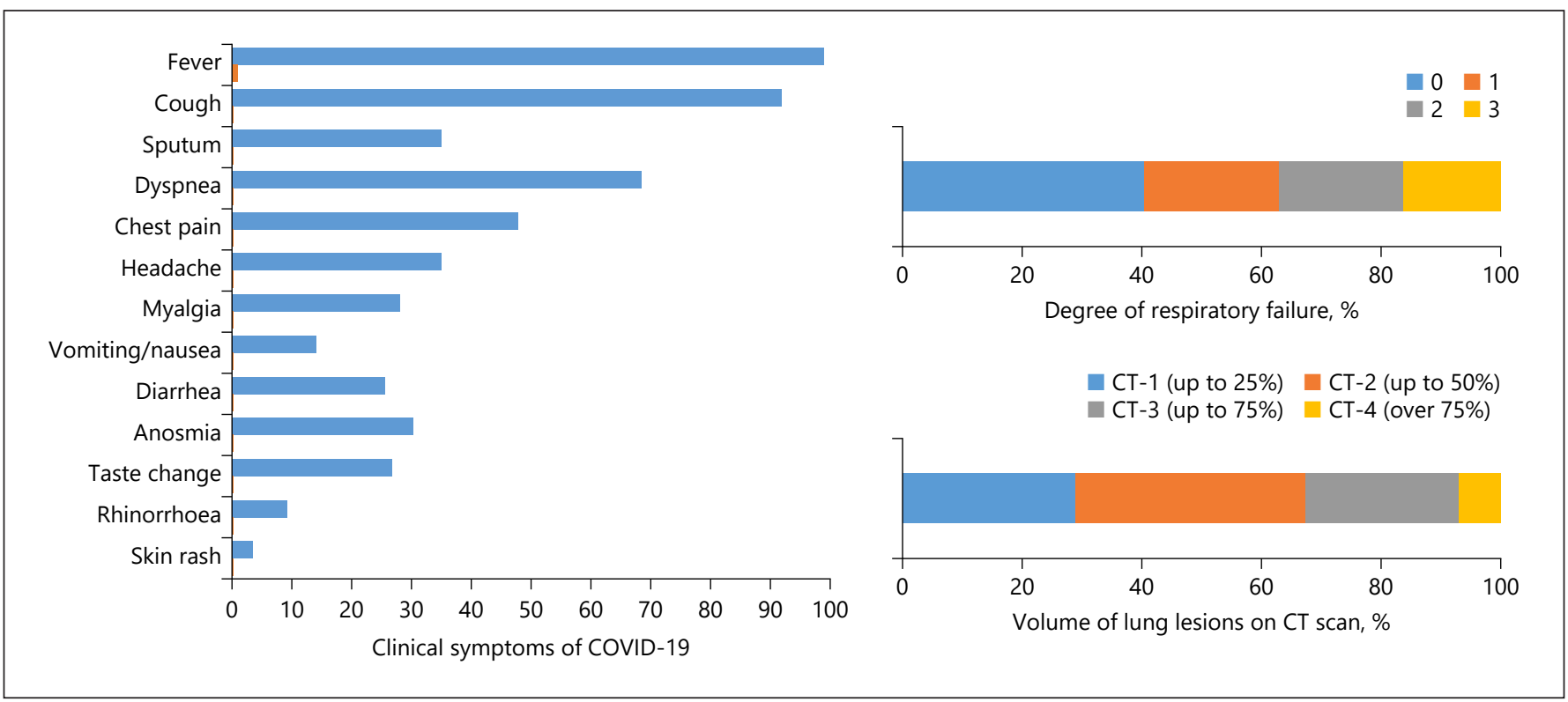

Fig. 1. Major pulmonary manifestations and extrapulmonary symptoms of COVID-19. CT-1 - lesion of lungs is under 25\%, CT-2 up to 50\%, CT-3 up to 75\%, and CT-4 over 75\%. COVID-19, coronavirus disease 2019.

Treatment of arrhythmia, chronic heart failure was carried out according to European Guidelines. It will be described in more detail in the section "Results." The average hospital period was 14 [10, 11] days. Death was considered as the primary endpoint.

\section{Statistical Analysis}

The material was statistically processed using SPSS Statistics 21. Quantitative signs are presented as $\mathrm{M} \pm$ (mean \pm one standard deviation) or as a median indicating the 1 st and 3 rd quartiles. Distribution normality was assessed using the Kolmogorov-Smirnov test. Significance of differences was assessed using the Student, Mann-Whitney, and Wilcoxon criteria. The differences were evaluated as significant at $p<0.05$. To estimate the correlations, the Spearman coefficient was determined. ROC analysis, multifactor analysis by linear logistic regression method, and Kaplan-Meier curves were used to evaluate prognostic significance of different parameters.

\section{Ethical Standards}

All patients have signed an informed consent to the examination and the various types of treatment for a new coronavirus infection.

\section{Results}

\section{General Clinical Characteristics of Patients}

Frequency of various COVID-19 symptoms, degree of respiratory failure, and stage of pneumonia according to $\mathrm{CT}$ are presented in Figure 1. Patients were admitted to the clinic on the 7 th day of the disease on average. Fever, cough, and shortness of breath were the most common. Anosmia and taste changes were more common in patients with moderate pneumonia. The volume of pneumonia by CT was different, but respiratory failure was present in almost $60 \%$ of the patients, which required oxygen therapy (Table 1). Glucose metabolic disorders and obesity were the most common background for the disease. The increase in laboratory markers of inflammation usually reflected the severity of the disease (see below). No significant elevation was found in the prepsin/procalcitonin levels.

\section{Cardiological Characteristics of Patients}

The total number of patients with cardiovascular disease and/or symptoms was 39 (45.3\%). More than a half of patients had hypertension of various degrees (Table 1). However, at the admission, high blood pressure was registered in rare patients. The number of patients with $\mathrm{CHD}$ history was low. Chest pain in almost half of the patients was not accompanied by ECG changes. Individual patients initially had chronic myocarditis with immunosuppressive therapy.

ECG changes, in addition to a few cases of postinfarction or myocarditis sclerosis (Fig. 2), consisted of flattened/negative $\mathrm{T}$ waves (mostly in lower leads), minimal ST-segment depression, and low-QRS voltage. Signs of left ventricular hypertrophy were found in $11 \%$. Atrial 
Table 1. Clinical characteristics of patients and outcomes of the disease

\begin{tabular}{|c|c|c|c|}
\hline Male/female (\%) & $48 / 38(56 / 44)$ & Arterial hypertonia $1 / 2 / 3$ degree & $8 / 19 / 22(57.0 \%)$ \\
\hline COVID-19 is confirmed by PCR, $n(\%)$ & $51(59)$ & CHF, $2 / 3$ NYHA class, $n(\%)$ & $6 / 2(9.3)$ \\
\hline Days of illness, upon admission & $7[6 ; 10]$ & $\mathrm{CHD} /$ myocardial infarct history & $15 / 11(17.4 / 12.8 \%)$ \\
\hline Peak body temperature, ${ }^{\circ} \mathrm{C}$ & $38.8[38.1 ; 39.2]$ & Stenting/bypass surgery history & $3 / 2(5.9 \%)$ \\
\hline Fever duration, days & $12[10 ; 14]$ & Chronic myocarditis, $n(\%)$ & $4(4.7)$ \\
\hline Diabetes/glucose tolerance disorder & $5 / 16(25 \%)$ & RFA history, $n(\%)$ & $2(2.3)$ \\
\hline Obesity $1 / 2 / 3$ degree & $29 / 7 / 3(45.3 \%)$ & $\mathrm{AF} /$ flutter & $14(16.9 \%) / 1(1.2 \%)$ \\
\hline $\mathrm{BMI}, \mathrm{kg} / \mathrm{m}^{2}$ & $29.2 \pm 5.8$ & AF at the time of hospitalization, $n(\%)$ & $8(9.3)$ \\
\hline Hyperlipidemia, $n(\%)$ & $32(37.2)$ & PSVBs, $n(\%)$ & $3(3.6)$ \\
\hline Leukocytes (min.), $\times 10^{9} / \mathrm{L}$ & $4.3[3.6 ; 5.3]$ & PVBs, $n(\%)$ & $5(6.2)$ \\
\hline Lymphocytes (min.), $\times 10^{9} / \mathrm{L}$ & $0.9[0.6 ; 1.2]$ & $\mathrm{LBBB} / \mathrm{RBBB}$ & $2 / 6(2.3 / 7.0 \%)$ \\
\hline $\mathrm{Cr}(\max ),. \mu \mathrm{mol} / \mathrm{L}$ & $102.0[88.5 ; 113.4]$ & Depression of ST, $n(\%)$ & $9(11.4)$ \\
\hline GFR, mL/min (SKD-EPI) & $66.5[53.8 ; 83.3]$ & LV EDD, $\mathrm{cm}$ & $4.8 \pm 0.8$ \\
\hline AST (max.), U/L & $58[36 ; 86]$ & LV EF, \% & $56[52.5 ; 62]$ \\
\hline ALT (max.), U/L & $64[35 ; 107]$ & Left/right atrium, $\mathrm{mL}$ & $63[51 ; 83] / 56[42 ; 71]$ \\
\hline $\mathrm{LDH}(\max ),. \mathrm{U} / \mathrm{L}$ & $615[496 ; 815]$ & Right ventricle, $\mathrm{cm}$ & $2.9[2.5 ; 3.5]$ \\
\hline $\mathrm{CK}(\max ),. \mathrm{U} / \mathrm{L}$ & $144[73 ; 301]$ & Systolic PA pressure, $\mathrm{mm} \mathrm{Hg}$ & $35[25 ; 38]$ \\
\hline Ferritin (max.), $\mu \mathrm{g} / \mathrm{L}$ & $498[336 ; 602]$ & Pericardial effusion & $\begin{array}{l}5(29.4 \% \\
\text { of those examined) }\end{array}$ \\
\hline Oxygen therapy (nasal), $n(\%)$ & $48(55.8)$ & Critical care unit, $n(\%)$ & $11(12.8)$ \\
\hline Oxygen therapy (by mask), $n(\%)$ & $10(11.6)$ & Invasive lung ventilation, $n(\%)$ & $8(9.3)$ \\
\hline Noninvasive lung ventilation, $n(\%)$ & $4(4.7)$ & Lethality, $n(\%)$ & $8(9.3)$ \\
\hline
\end{tabular}

CRP, C-reactive protein; AF, atrial fibrillation; LDH, lactate dehydrogenase; CK, Cr kinase; COVID-19, coronavirus disease 2019; $\mathrm{CHF}$, chronic heart failure.

fibrillation (AF) was diagnosed in 17\%, including 8 patients at the admission (Fig. 2). In one case, AF was registered for the first time, as well as supraventricular extrasystole in 3 patients. Ventricular arrhythmias were present in history of 5 patients, but they were registered at the clinic only in one case. The right bundle branch block was the most frequent conduction disturbance. Four patients had a pacemaker, and 2 patients had previously performed RFA (for AF and supraventricular tachycardia) without effect.

In most cases, patients were not fully examined before. It is not possible to determine the duration of the arrhythmia. Troponin levels were studied in patients with cardiac symptoms and/or severe pneumonia and only in 2 cases showed a slight increase. In EchoCG, the mean parameters were within the normal range, except for a slight increase of systolic pulmonary artery pressure, Table 1.
Almost $30 \%$ of the examined patients showed pericardial effusion, which was not correlated with the presence of congestion and was considered as manifestation of pericarditis.

\section{The Level of Anti-Heart Antibodies and Its Correlation with Clinical Data}

In general, the titers of 2 and more types of antibodies were increased by 3 and more times in 25 (73.5\%) of the screened patients. Depending on the level of AHA and their correlation with the overall disease activity, several subgroups can be identified. In $29 \%$ of the examined patients, high titers of AHA were detected in the presence of high immune-inflammatory activity of COVID-19. Another $12 \%$ of patients with high immuno-inflammatory activity showed only moderate increase in AHA titers. In $21 \%$ of patients, high AHA titers were noted despite 

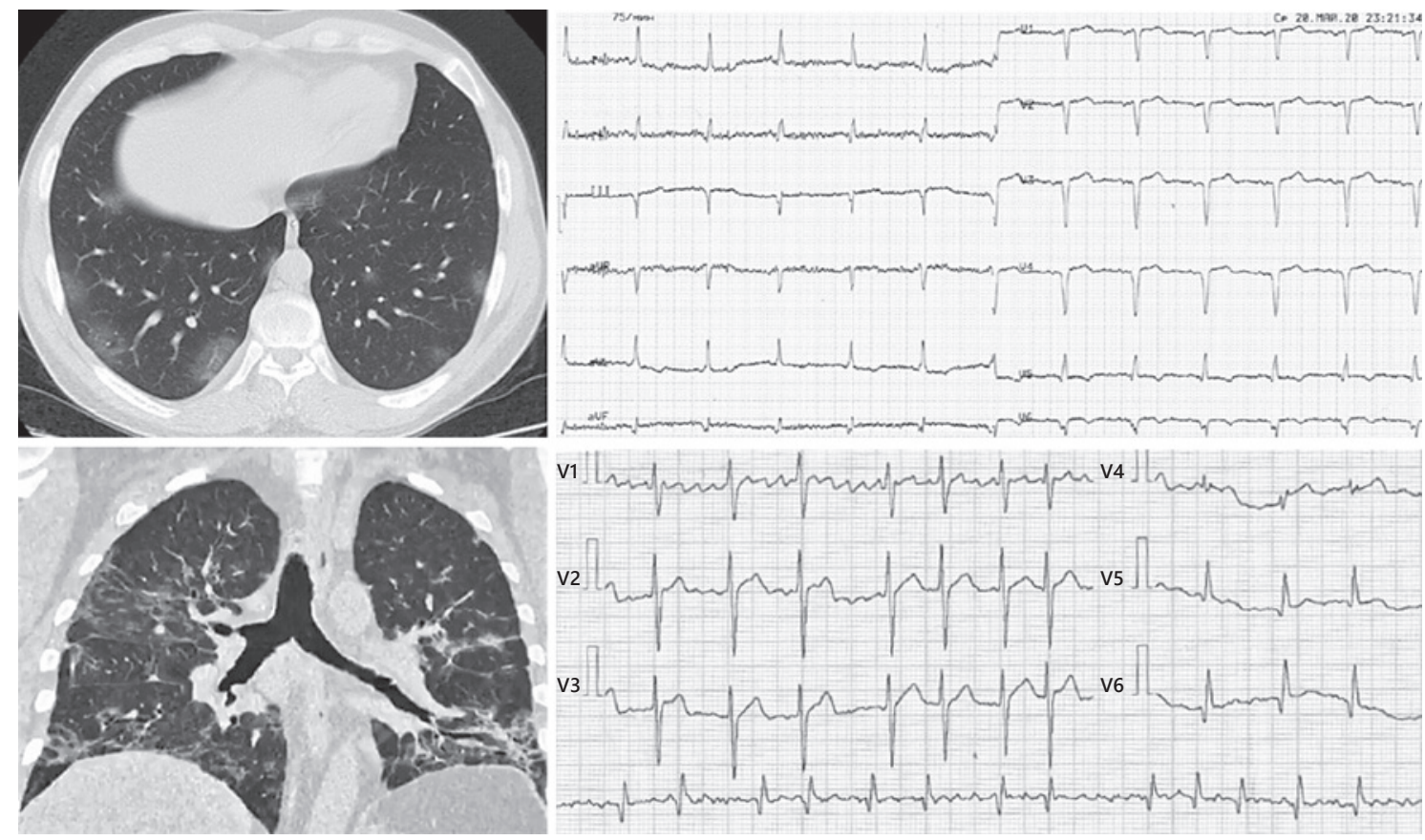

Fig. 2. Examples of CT and ECG changes in patients with COVID-19. On the left is chest CT (CT-1 in the upper figure and CT-3 in the lower figure). On the right is the ECG, speed $25 \mathrm{~mm} / \mathrm{s}$. On the top - myocardial scarring in a patient with a previous infarct-like myocarditis, on the bottom - AF recorded at admission to the clinic. AF, atrial fibrillation; COVID-19, coronavirus disease 2019; ECG,electrocardiography.

low COVID-19 activity. In $26 \%$ of patients, there was no increase in both general immuno-inflammatory markers and AHA titer. Finally, 4 patients (12\% of examined for AHA patients) suffered from chronic myocarditis and received maintenance immunosuppressive therapy by the time of coronavirus pneumonia; no significant increase in AHA was detected among them.

The following significant correlations of AHA were found: (1) antibodies to cardiomyocyte nuclei antigens with cardiovascular manifestations $(r=0.459, p<0.01)$ and pericardial effusion $(r=0.721, p<0.05)$. (2) Antibodies to cardiomyocyte antigens - with severity of pneumonia $(r=0.472, p<0.05)$, dyspnea $(r=0.370, p<0.05)$, respiratory failure $(r=0.387, p<0.05)$, the need for oxygen therapy $(r=0.388, p<0.05)$, to use a mask $(r=0.540$, $p<0.01)$ and invasive lungs ventilation $(r=0.469, p<$ $0.01)$, the presence of chest pain $(r=0.374, p<0.05)$, low voltage of QRS complexes $(r=0.415, p<0.05)$, pericardial effusion $(r=0.745, p<0.05)$, maximum level of CRR $(r=0.360, p<0.05)$, and LDH $(r=0.360, p<0.05)$. (3) Antibodies to smooth muscle antigens - with respiratory failure $(r=0.343, p<0.05)$, the need for oxygen therapy $(r=0.423, p<0.05)$, and the AF at admission $(r=0.414$, $p<0.05)$.

\section{Therapy and Outcomes}

Cardiotropic, anticoagulant therapy, and treatment of coronavirus pneumonia are presented in Table 2. All patients without exception received anticoagulant therapy, mainly low-molecular-weight heparins in various doses (from therapeutic to prophylactic). New oral anticoagulants were prescribed to patients with AF or intracardiac thrombosis in their history. The basic treatment for coronavirus pneumonia was a combination of hydroxychloroquine and azithromycin.

Clarithromycin, ceftriaxone, levofloxacin, and other antibacterial agents were used as an alternative for severe pneumonia. Lopinavir-ritonavir was prescribed to a few patients. In severe course of COVID-19 with cytokine storm, interleukin-6 inhibitors as well as corticosteroids (dexamethasone 4-8 mg/day) were used. Methylprednisolone maintenance therapy continued in patients with chronic myocarditis. Cardiotropic drugs were administrated according to standard indications. 
Table 2. Medical treatment of patient with COVID-19

\begin{tabular}{lllc}
\hline Drugs & $\begin{array}{l}\text { Frequency of } \\
\text { prescription, \% }\end{array}$ & Drugs & $\begin{array}{l}\text { Frequency of } \\
\text { prescription, \% }\end{array}$ \\
\hline Low-molecular-weight heparins & 83.7 & Levofloxacin & 26.0 \\
New oral anticoagulants & 16.3 & Ceftriaxone & 27.0 \\
$\beta$-Blockers & 37.2 & Clarithromycin & 12.8 \\
ACE inhibitors & 29.1 & Azithromycin & 83.7 \\
Angiotensin II receptor blockers & 11.6 & Other antibacterial agents & 14.0 \\
Antiarrhythmic drugs IC & 3.5 & Hydroxychloroquine & 93.0 \\
Amiodarone & 4.7 & Methylprednisolone & 4.7 \\
Spironolactone & 8.1 & Dexamethasone & 12.6 \\
Loop diuretics & 10.5 & Tocilizumab/sarilumab & $4.7 / 1.2$ \\
Acetylsalicylic acid & 12.8 & Lopinavir + ritonavir & 7.0 \\
\hline
\end{tabular}

COVID-19, coronavirus disease 2019.

Eleven patients were admitted to the intensive care unit (see Table 1). The main indications were progressive respiratory failure, ineffectiveness of oxygen therapy, and noninvasive ventilation. Invasive ventilation of the lungs was performed in 8 patients. Lethality was $9.3 \%$ (8 patients). The causes of death were respiratory failure (in all cases) in combination with cardiac failure $(n=3)$, renal failure $(n=4)$, pulmonary embolism verified in autopsy $(n=2)$, and sepsis $(n=2)$.

\section{Prognostic Significance of Cardiovascular Diseases/ \\ Symptoms in Comparison with Other Factors}

Any cardiovascular disease/symptoms had a prognostic value in relation to death (Fig. 3). A significant difference in the frequency of lethal outcomes in patients with and without cardiac injury has been revealed. The D-dimer was most closely related to the heart involvement. In the presence of cardiovascular diseases, the maximum $\mathrm{D}$ dimer level was significantly higher than without it (1.30 $[0.62 ; 4.39]$ compared to $0.46[0.32 ; 0.93] \mu \mathrm{g} / \mathrm{mL}, p=$ $0.002)$. D-dimer level $>0.5 \mu \mathrm{g} / \mathrm{mL}$ had a sensitivity of $79.2 \%$ and specificity of $60 \%$ in the prediction of cardiovascular manifestations. The D-dimer over $1 \mu \mathrm{g} / \mathrm{mL}$ had specificity $90 \%$ (with decreasing sensitivity). No other clinical or laboratory marker of disease activity correlated so much with cardiac injury.

Maximum levels of D-dimer, CRR, LDH, severity of dyspnea, and volume of oxygen therapy were the most important factors of prognosis. The age of 73 years and older was a predictor of lethal outcome with a sensitivity of $87.5 \%$ and specificity of $85.1 \%$, the need for oxygen therapy with flow speed of $9 \mathrm{~L} / \mathrm{min}$ and above -100 and $85.7 \%$, the maximum number of leukocytes from 7.0 thousand and more -87.5 and $75.0 \%$, maximum level of CRR over $125 \mathrm{mg} / \mathrm{L}-100$ and 82.3\%, D-dimer over 1.7 $\mathrm{mg} / \mathrm{mL}-100$ and $89.1 \%$, LDH over $850 \mathrm{U} / \mathrm{L}-100$ and $98.9 \%$, CK over $800 \mathrm{U} / \mathrm{L}-83.3$ and $87.7 \%$, aspartate aminotransferase over $75 \mathrm{U} / \mathrm{L}-85.7$ and $73.4 \%$, and Cr over $112 \mu \mathrm{g} / \mathrm{L}-85.7$ and $77.9 \%$, respectively.

The level of various AHA directly correlated with lethality (for antibodies to cardiomyocytes $r=0.363$ and for antibodies to smooth muscle antigens $r=0.426, p<0.05$ ). Prognostic significance of antibodies to smooth muscle antigens confirmed by ROC analysis (AUC $0.887, p<$ $0.05)$.

In the multifactor analysis by linear regression method, the model with inclusion of 6 factors - age, diabetes, oxygen therapy volume, maximum leukocyte level, CRP, and D-dimer (correlation coefficient 0.871, corrected square of correlation coefficient $0.726, p<0.001$ ) - showed the greatest prognostic significance. Adding to this model, the presence of cardiovascular disease or symptoms (regardless of the connection with coronavirus infection) does not enhance the predictive value. However, a model involving risk factors not directly related to the severity of pneumonia - age, diabetes, and cardiovascular disease/ symptoms - also has high predictive value (correlation coefficient $0.568, p<0.001$ ).

\section{Discussion}

The analysis of one of the Moscow University registers of patients with coronavirus pneumonia confirmed the high frequency of cardiovascular pathology and its prognostic importance. The proportion of patients with car- 


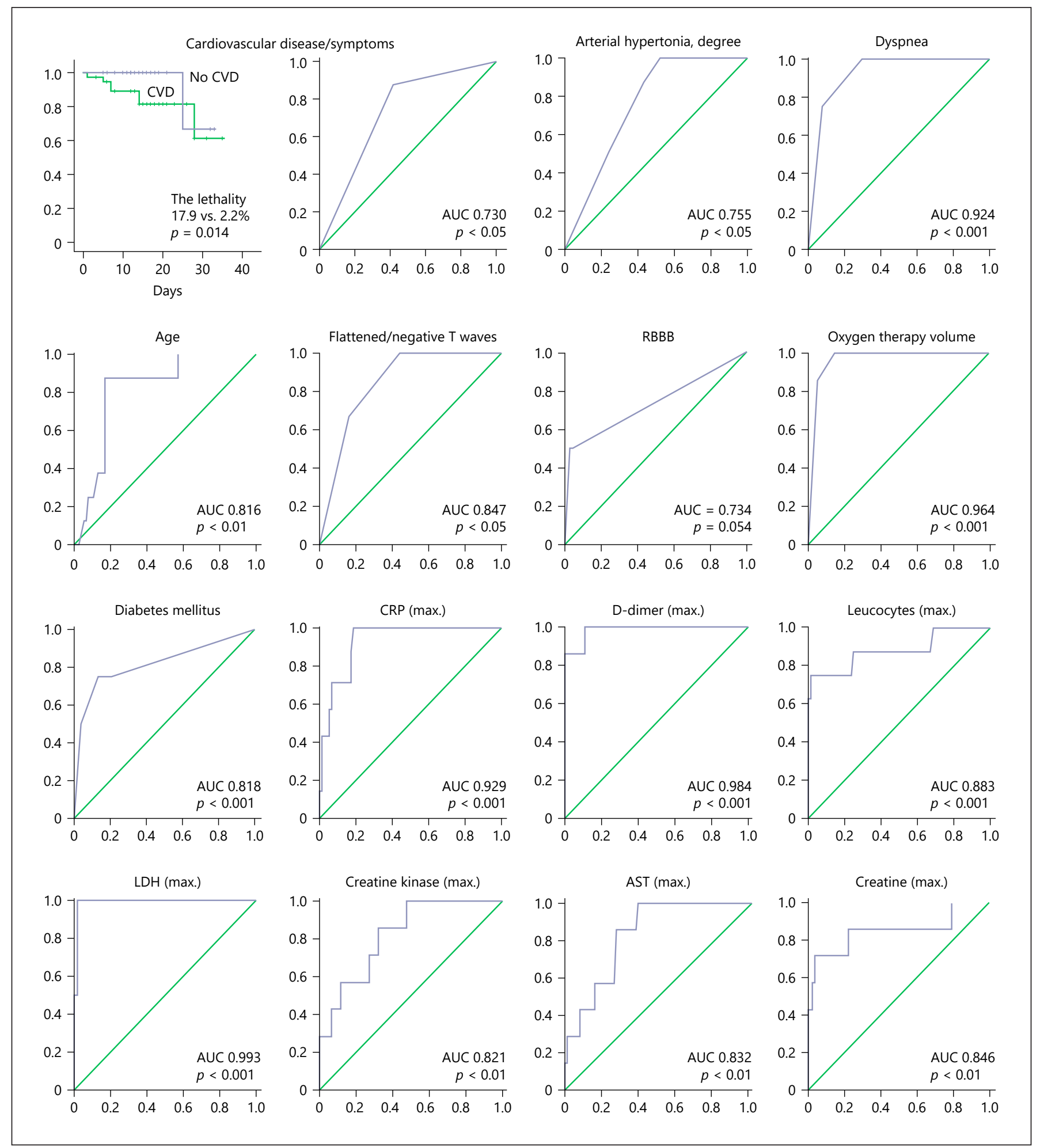

Fig. 3. Prognostic significance of cardiovascular disease/symptoms and other factors in predicting death in hospital patients with coronavirus pneumonia. Kaplan-Meyer survival curves (first chart) and ROC analysis (other charts) results. AUC, area under curve; $p$, statistical significance; RBBB, right bundle branch block; CRP, C-reactive protein; LDH, lactate dehydrogenase; AST, aspartate aminotransferase.

Cardiovascular Diseases in Coronavirus Pneumonia
Int Arch Allergy Immunol 2021;182:765-774 DOI: $10.1159 / 000515253$ 
diovascular manifestations was high (45.3\%), but in most cases, it was hypertension. The most significant marker of viral myocardial injury considered troponin increase, which was found in $8 \%$ of patients [12]. In our study, troponin levels were determined only in clinically suspicious patients and remained normal in most cases. The acute myocardial injury can be considered in patients with arrhythmias, the duration of which was most often unknown.

Among the rhythm disorders, the supraventricular arrhythmia was predominated. It corresponds to the larger study in which supraventricular arrhythmias were detected in $26.4 \%$ of 915 patients [13]. Despite the high frequency of hydroxychloroquine prescription, we have not observed life-threatening ventricular arrhythmias. We did not obtain a direct correlation of arrhythmias with the inflammatory activity. A strong correlation with the size of both atria $(r=0.7-0.8)$ was revealed. It is suggested that the lack of correlation of arrhythmia with pneumonia severity indicates in favor of COVID myocarditis [10].

The true frequency of myocarditis without biopsy is unclear. There are only few reports about lifetime proved lymphocytic and eosinophilic myocarditis in COVID-19 $[14,15]$, including positive PCR results on SARS-CoV-2 RNA [16]. We showed the lymphocytic myo-, endo- and pericarditis in autopsy patients with COVID-19 [17]. The present register includes one of such patients, and data from other autopsies are not fully available. However, myocarditis can be clinically indicated not only by arrhythmias, but also by repolarization disorders, low-QRS voltage on ECG, significant increase of AHA level, as well as pericardial effusion (it confirmed by the positive effect of steroids). We can also suppose the relationship between atrial rhythm disorders and pericarditis. However, pericarditis clearly correlated with the disease activity the degree of the fever, the level of inflammatory markers, as well as the severity of pneumonia by CT $(r=0.64)$. Autopsy studies showed the lymphocytic nature of pericarditis [17], typical both for reactive effusion and for independent viral-immune affection.

The increase in AHA titers revealed in 3/4 patients clearly correlated both with the general immune-inflammatory activity, severity of pneumonia and with signs of myocardial/pericardial lesions. However, elevated AHA titers were not obligatory associated with the severity of coronavirus pneumonia and the degree of overall laboratory activity. Some patients with severe pneumonia did not have significantly elevated AHA titers, while others had significantly elevated AHA titers in spite of low pneumonia activity. These facts suggest a certain selectivity and specificity of autoimmune reaction to cardiac antigens in patients with COVID-19. It can be suggested that latent chronic myocarditis in some patients preceded the development of coronavirus infection. In this case, the increase in AHA reflects the previous inflammatory reaction or hypersensitivity of previously sensitized myocardium to SARS-CoV-2.

On the other hand, the absence of AHA in a part of patients with severe pneumonia suggests that direct or mediated (due to ischemia, oxidative stress, etc.) myocardial injury with exposure of its antigens is probably necessary for AHA production. Mechanisms of myocardial protection or increased vulnerability to SARS-CoV-2 require investigation. We can suggest an important pathogenetic role of AHA in the development of myocardial injury. The identified correlations of ANA with cardiac symptoms (e.g., arrhythmias and pericardial effusion) support this hypothesis. The key question seems to be the role of AHA in the further development of the disease. We can expect both the decline of their production due to regression of the hyperinflammatory response and its involvement in maintaining chronic myocarditis.

In addition, we found a strong correlation between maximum D-dimer levels and cardiovascular disease/ symptoms, which indicates a role of coronavirus-specific prothrombogenic mechanisms. The high level of D-dimer is typical for coronavirus pneumonia in comparison with other pneumonia [18]. It is closely related not only to the total disease severity [11] and lethality (threshold value $2 \mu \mathrm{g} / \mathrm{mL}$ in comparison with our $1.7 \mu \mathrm{g} / \mathrm{mL}$ [19]), but also with acute pulmonary artery thromboembolism [20], deep veins thrombosis [21], liver damage [22], and neurological manifestations [23]. According to ROC analysis, the predicted value of $\mathrm{D}$-dimer in respect to organ damage was 0.889 [11].

But association of cardiovascular manifestations with higher D-dimer level is shown only in few reports [5]. In patients with ST elevation, there was a higher D-dimer level in cases of myocardial infarction compared to patients without major artery lesions [24]. In our study, there were no patients with verified large coronary artery thrombosis. The D-dimer level, which corresponds to increase of cardiac manifestations, was $0.5-1.0 \mu \mathrm{g} / \mathrm{dL}$. The role of microthrombosis of small coronary arteries in the various cardiac symptoms can be confidently concluded.

Other factors associated with cardiac injury in COVID-19 include severe pneumonia, increased levels of ESR, CRP, fibrinogen, alaninaminotransferase, $\mathrm{LDH}$, interleukin-6, and the precursor of amyloid SAA [5]. In our study, these factors have not shown a role in cardiac in- 
jury. But their influence on the lethality was revealed. The model with 6 factors - age, diabetes, oxygen therapy volume, maximum leukocyte level, CRP, and D-dimer showed the greatest prognostic importance. They reflect the key pathogenic mechanisms of the disease (hyperinflammation, prothrombogenicity, and immunodeficiency with the risk of secondary bacterial infections), its leading clinical manifestation (respiratory failure), and aggravating factors (age and diabetes).

However, the same fact of the cardiovascular lesion, as well as separately - arterial hypertension in the history (or first identified) had a major impact on the prognosis. In an early publication, the cause of lethal outcomes in $7 \%$ of 68 patients was myocardial injury and in $33 \%$ - its combination with respiratory failure [7]. In a cohort of 416 patients, mortality in the presence of cardiac injury was $51.2 \%$ in comparison with $4.5 \%$ without it $(p<0.001$ [6]). The analysis of $>72,000$ cases confirmed these conclusions: the general mortality was $2.3 \%$, in hypertensive patients this index increased up to $6.0 \%$ and in cardiovascular pathology in general - up to $10.5 \%$ [8]. Thus, the presence of cardiovascular disease and symptoms has taken place among the unfavorable prognostic factors in patients with coronavirus pneumonia, regardless of the presence of their pathogenetic relationship with the infection itself.

\section{Conclusions}

The incidence of cardiovascular disease and symptoms in COVID-19 is 45.3\%. Arrhythmias, heart failure, low-QRS voltage, and pericardial effusion are the typical manifestations. An increase in AHA was observed in 73.5\%. It may be part of the systemic immune and inflammatory response and play direct role in the heart injury. The cardiovascular pathology correlated with the maximum D-dimer level. Cardiac failure was one of the causes of death in 3 out of 8 patients (37.5\%). Lethality in the presence of cardiovascular pathology was significantly higher than without it ( 17.9 vs. $2.2 \%, p<0.05)$. The most powerful prognostic model for adverse outcomes of COVID-19 includes age, diabetes, oxygen therapy volume, maximum leukocyte level, CRP, and D-dimer.

\section{Acknowledgements}

The authors of the article bring sincere gratitude to all doctors of the department for the treatment of new coronavirus infection No. 5 (M.M. Beraya, I.V. Novikova, A.V. Sedov, D.A. Tsaregorodtsev, P.A. Sheluha, and I.G. Yudin) and other infectious disease

Cardiovascular Diseases in Coronavirus Pneumonia departments, doctors of radiation and functional diagnostics of UKB N1 COVID-19 of the Sechenov University Hospital, who took direct part in the examination and treatment of patients included in this study.

\section{Statement of Ethics}

All procedures performed in studies involving human participants were in accordance with the ethical standards of the institutional research committee and with the 1964 Helsinki declaration and its later amendments or comparable ethical standards. All patients signed an informed consent form for all the examinations and treatment. There was ethical permit of University (No. 0353).

\section{Conflict of Interest Statement}

All the authors declare no conflicts of interest.

\section{Funding Sources}

The authors did not receive any funding.

\section{Author Contributions}

Blagova O.V. - study idea, data collection, statistical processing, and text writing. Varionchik N.V. - data collection, statistical processing, and text writing. Zaidenov V.A. - laboratory tests. Savina P.O. - data collection. Sarkisova N.D. - general management of patient care and research.

References

1 Mehta P, McAuley DF, Brown M, Sanchez E, Tattersall RS, Manson JJ, et al. COVID-19: consider cytokine storm syndromes and immunosuppression. Lancet. 2020;395(10229): 1033-4.

2 Huang C, Wang Y, Li X, Ren L, Zhao J, Hu Y, et al. Clinical features of patients infected with 2019 novel coronavirus in Wuhan, China. Lancet. 2020 Jan 30;395(10223):497-506.

3 Qin C, Zhou L, Hu Z, S, S, Y, et al. Dysregulation of immune response in patients with COVID-19 in Wuhan, China. Clin Infect Dis. 2020;71(15):762-8

4 Zheng YY, Ma YT, Zhang JY, Xie X. COVID-19 and the cardiovascular system. Nat Rev Cardiol. 2020;17(5):259-60.

5 Li M, Dong Y, Wang H, Guo W, Zhou H, Zhang Z, et al. Cardiovascular disease potentially contributes to the progression and poor prognosis of COVID-19. Nutr Metab Cardiovasc Dis. 2020;30(7):1061-7. 
6 Shi S, Qin M, Shen B, Cai Y, Liu T, Yang F, et al. Association of cardiac injury with mortality in hospitalized patients with COVID-19 in Wuhan, China. JAMA Cardiol. 2020;5(7): $802-10$

7 Ruan Q, Yang K, Wang W, Jiang L, Song J. Clinical predictors of mortality due to COVID-19 based on an analysis of data of 150 patients from Wuhan, China. Intensive Care Med. 2020 Apr 6;46(5):846-8.

$8 \mathrm{Wu} \mathrm{Z,} \mathrm{McGoogan} \mathrm{JM.} \mathrm{Characteristics} \mathrm{of} \mathrm{and}$ important lessons from the coronavirus disease 2019 (COVID-19) outbreak in China: summary of a report of 72,314 cases from the chinese center for disease control and prevention. JAMA. 2020;323(13):1239-42.

9 Van Linthout S, Klingel K, Tschöpe C. SARS$\mathrm{CoV}$-2-related myocarditis-like syndromes Shakespeare's question: what's in a name? Eur J Heart Fail. 2020;22(6):922-5.

10 Kochav SM, Coromilas E, Nalbandian A, Ranard LS, Gupta A, Chung MK, et al. Cardiac arrhythmias in COVID-19 infection. Circ Arrhythm Electrophysiol. 2020;13(6):e008719.

11 Xu Y, Qian Y, Gu Q, Tang J. Relationship between D-dimer concentration and inflammatory factors or organ function in patients with coronavirus disease 2019. Zhonghua Wei Zhong Bing Ji Jiu Yi Xue. 2020;32(5):559-63.

12 Wang D, Hu B, Hu C, Zhu F, Liu X, Zhang J, et al. Clinical characteristics of 138 hospitalized patients with 2019 novel coronavirus-infected pneumonia in Wuhan, China. JAMA. 2020;323(11):1061-9.
13 Gopinathannair R, Merchant FM, Lakkireddy DR, SP, S, JK, et al. COVID-19 and cardiac arrhythmias: a global perspective on arrhythmia characteristics and management strategies. J Interv Card Electrophysiol. 2020;59(2): 329-36.

14 Sala S, Peretto G, Gramegna M, Palmisano A, Villatore A, Vignale D, et al. Acute myocarditis presenting as a reverse Tako-Tsubo syndrome in a patient with SARS-CoV-2 respiratory infection. Eur Heart J. 2020;41(19):18612.

15 Craver R, Huber S, Sandomirsky M, McKenna D, Schieffelin J, Finger L. Fatal eosinophilic myocarditis in a healthy 17 -year-old male with severe acute respiratory syndrome coronavirus 2 (SARS-CoV-2c). Fetal Pediatr Pathol. 2020;1-6.

16 Escher F, Pietsch H, Aleshcheva G, Bock T, Baumeier C, Elsaesser A, et al. Detection of viral SARS-CoV-2 genomes and histopathological changes in endomyocardial biopsies. ESC Heart Fail. 2020 Oct;7(5):2440-7.

17 Kogan EA, Berezovskiy YS, Blagova OV, Kukleva $\mathrm{AD}$, Bogacheva GA, Kurilina EV, et al. [Miocarditis in patients with COVID-19 confirmed by immunohistochemical]. Kardiologiia. 2020;60(7):4-10.

18 Yin S, Huang M, Li D, Tang N. Difference of coagulation features between severe pneumonia induced by SARS-CoV2 and non-SARSCoV2. J Thromb Thrombolysis. 2020;1-4.
19 Zhang L, Yan X, Fan Q, Liu H, Liu X, Liu Z, et al. D-dimer levels on admission to predict in-hospital mortality in patients with Covid-19. J Thromb Haemost. 2020;18(6):13249.

20 Leonard-Lorant I, Delabranche X, Severac F, Helms J, Pauzet C, Collange O, et al. Acute pulmonary embolism in COVID-19 patients on CT angiography and relationship to Ddimer levels. Radiology. 2020;296(3):E18991.

21 Artifoni M, Danic G, Gautier G, Gicquel P, Boutoille D, Raffi F, et al. Systematic assessment of venous thromboembolism in COVID-19 patients receiving thromboprophylaxis: incidence and role of $\mathrm{D}$-dimer as predictive factors. J Thromb Thrombolysis. 2020; 50(1):211-6.

22 Yao N, Wang SN, Lian JQ, YT, GF, WZ, et al. Clinical characteristics and influencing factors of patients with novel coronavirus pneumonia combined with liver injury in Shaanxi region. Zhonghua Gan Zang Bing Za Zhi. 2020;28(3):234-9.

23 Ellul MA, Benjamin L, Singh B, Lant S, Michael BD, Easton A, et al. Neurological associations of COVID-19. Lancet Neurol. 2020; 19(9):767-83.

24 Bangalore S, Sharma A, Slotwiner A, Yatskar L, Harari R, Shah B, et al. ST-segment elevation in patients with Covid-19: a case series. N Engl J Med. 2020;382(25):2478-80. 